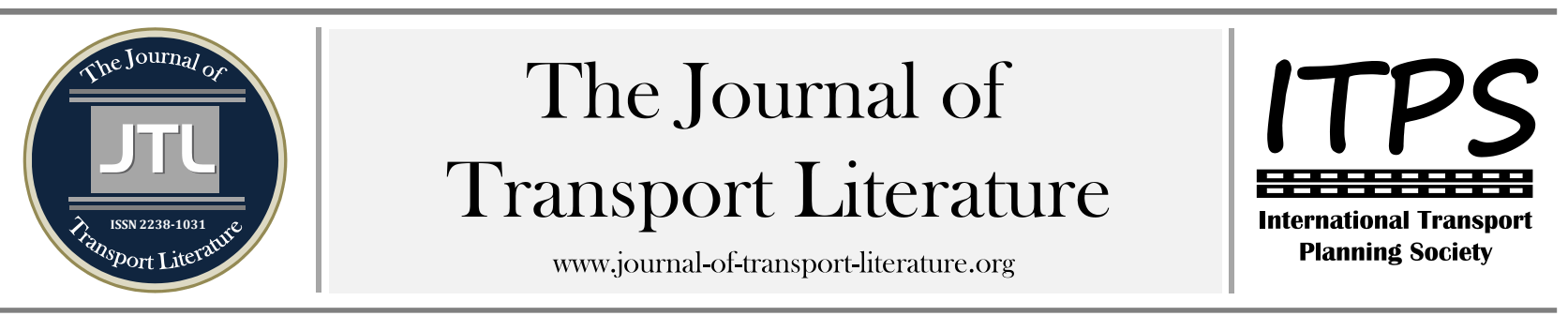

\title{
A multiobjective metaheuristic approach for the integrated vehicle and crew scheduling
}

Bruno de Athayde Prata ${ }^{+}$

Universidade Federal do Ceará, Fortaleza, Brasil

\section{Article Info}

Keywords:

mass transit

combinatorial optimization

evolutionary algorithms

Submitted 18 Feb 2015:

received in revised form 21 May

2015; accepted 12 Jul 2015

Licensed under

Creative Commons

CC-BY 3.0 BR.

\section{Abstract}

The integrated vehicle and crew scheduling problem is a hard Combinatorial Optimization problem widely studied over the years. Taking into consideration the range of variables related to the planning process of vehicles and drivers, there are several practical characteristics of the problem that are not reflected in the solutions generated computationally. Among these characteristics, that were not found in the consulted literature, the most important is the existence of multiple objectives. This paper aims at presenting a multiobjective approach for the integrated vehicle and crew scheduling problem based on Genetic Algorithms. A case study in Portimão (Portugal) is presented and discussed. Were applied: (i) a Pareto Envelope-based Selection Algorithm II (PESA-II), and (ii) a hybridization between PESA-II and Integer Programming, which were summarized in a table. These results indicate that this new approach has a considerable potential for achieving significant gains in terms of operation costs and reduction in planning times.

+ Corresponding author. Universidade Federal do Ceará, Centro de Tecnologia. Campus do Picí, Bloco 710. Pici. 60455760 - Fortaleza, CE - Brasil.

E-mail address: baprata@ufc.br.

\section{Introduction}

In what concerns buses, urban transportation planning can be divided in several steps, often considered as follows: network design, timetable creation, vehicle scheduling, crew scheduling and crew rostering. Vehicle scheduling and crew scheduling have been a subject of numerous research activities, due to the significant gains that may result from their optimization (Daduna and Paixão, 1995; Wren and Rousseau, 1999).

Several researchers have reported the strong interaction between the vehicle scheduling problem and the crew scheduling problem, emphasizing the gains that can be obtained by considering them together (Freling et al., 2003). The integrated problem is usually known as the Vehicle and Crew Scheduling Problem (VCSP).

Traditionally, vehicle scheduling and crew scheduling are done separately and in a sequential manner. The Vehicle Scheduling Problem (VSP) is performed in a first stage, being followed by the Crew Scheduling Problem (CSP). Usually, the CSP is more complex than the VSP, and therefore, in general, it is not a suitable strategy to schedule the vehicles without taking into account the requirements for the crews, since these may form the bottleneck of the whole process.

In many public transport companies, particularly in developing countries, the costs of crews and drivers are the most significant. Thus, efforts should be focused in minimizing such costs, even when vehicle scheduling is being performed.

For the resolution of the VCSP, several approaches have been reported in the literature. Exact optimization methods, based on branch-and-bound, branch-and-cut, column generation, or lagrangian relaxation are presented by Friberg and Haase (1999), Gaffi and Nonato (1999), Haase et al. (2001), Freling et al. (1999), Freling et al. (2001), Fischetti et al. (2001), Freling et al. (2003), Huisman et al. (2005), Groot and Huisman (2008) and Mesquita and Paias (2008, 2009).

Approaches based on heuristics and metaheuristics are presented in Ball et al. (1983), Falkner and Ryan (1992), Patrikalakis and Xerocostas (1992), Wren and Gualda (1999), Valouxis and Housos (2002), Rodrigues et al. (2006), Laurent and Hao (2007), Steinzen et al. (2007) and Bartodziej et al. (2009). However, the reported heuristic techniques are not easy to generalize and most of them have been developed for very specific problems, with results reported for a small number of instances.

Taking into consideration the range of variables related to the planning process of vehicles and drivers, there are several practical characteristics of the problem that are not reflected in the solutions generated computationally. Among these characteristics, that were not found in the consulted literature, the most important is the existence of multiple objectives.

This paper aims at presenting a new multiobjective metaheuristic approach for the VCSP, based on the Pareto Envelopebased Selection Algorithm II (PESA-II).

This paper is structured as follows: the problem statement is described in the first section. In the second section, the model proposed is applied and the results are presented. The third section presents some conclusions and suggestions for future research. 


\section{Problem Statement}

In the model proposed here, the duties are generated directly over the trips and not over vehicle blocks previously defined, as it happens in traditional sequential approaches. Based on a trip timetable and on a set of labor constraints, crew member duties are generated, in order to produce a duty matrix ( $A$ matrix).

Based also on the same timetable, vehicles are scheduled, to perform the trips. Therefore, crew member scheduling will not be directly linked to vehicle scheduling, just as it happens in the sequential approaches. Both schedules (for vehicles and crews) are "related" to the timetable. Our main goal, then, is to cover the trips with available resources (vehicles and crew members). So, the problem can be viewed as a "multiple resource covering problem". This model is here referred as the Maximal Covering Problem with Multiple Resources (MCPMR).

Let $T$ a set of trips defined by a timetable, so that $T=M$. The relationship $i \alpha k$ represents the compatibility between trips $i$ and $k$, i.e., the trip $k$ can be made right after the trip $i$ by the same vehicle ( $c_{i k}$ below is the modeling parameter for this possibility).

Maximize $f_{1}=\sum_{i=1}^{m} \sum_{k=1}^{m} c_{i k} y_{i k}-\sum_{i=1}^{m} z_{i}$

Minimize $f_{2}=\sum_{i=1}^{n} t_{i}$

Subject to:

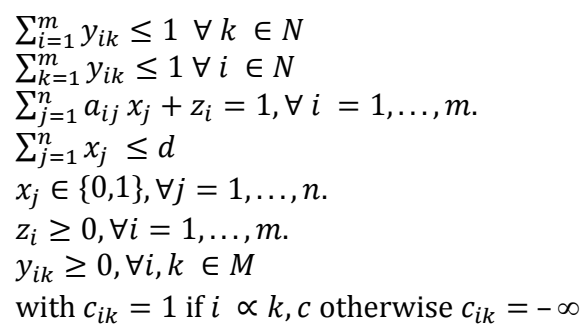

The objective function (1) maximizes the number of covered pieces-of-work. The first term is related to the covering of trips by vehicles and the second term is related to the covering of trips by crew duties. The objective function (2) minimizes the idle times of the vehicles between two consecutive trips. Constraints (3) and (4) impose that one vehicle covers one trip only once. Since the coefficient matrix of the constraints of an assignment problem is unimodular, the integrality of the variables $y_{i k}$ can be relaxed. If the leg $i$ cannot be covered, constraints (5) impose that $z_{i}=1$. As a result of these constraints, the integrality of variables $z_{i}$ can be relaxed. Constraint (6) imposes that no more than $d$ columns from matrix $A$ are selected to be included in the solution. Finally, constraints (7), (8), and (9) define the scope of the model variables.

\section{Proposed approach}

The PESA algorithm, proposed for Corne et al. (2000), consists in an evolutionary metaheuristic based on the concept of hypergrid. A hypergrid is a grid in hyperplane, which aims to control the density of solutions in a normalized space of objectives. This mechanism has great importance to the efficiency of selection operators, as well as to maintain the diversity of the population. Corne et al. (2001) extended the cells' density concept like a criteria for the quality of solutions, creating the PESA-II algorithm. In this approach, applying the selection operator, for instance, the binary tournament, a sparse cell has a greater probability to be selected than a dense cell. Selecting a given cell, one of its solutions is randomly selected and the other genetic operators are performed.

The proposed algorithm is based on PESA-II evolutionary metaheuristic. As follows, in Figure 1, is presented the proposed approach for resolution of the multiobjective VCSP.

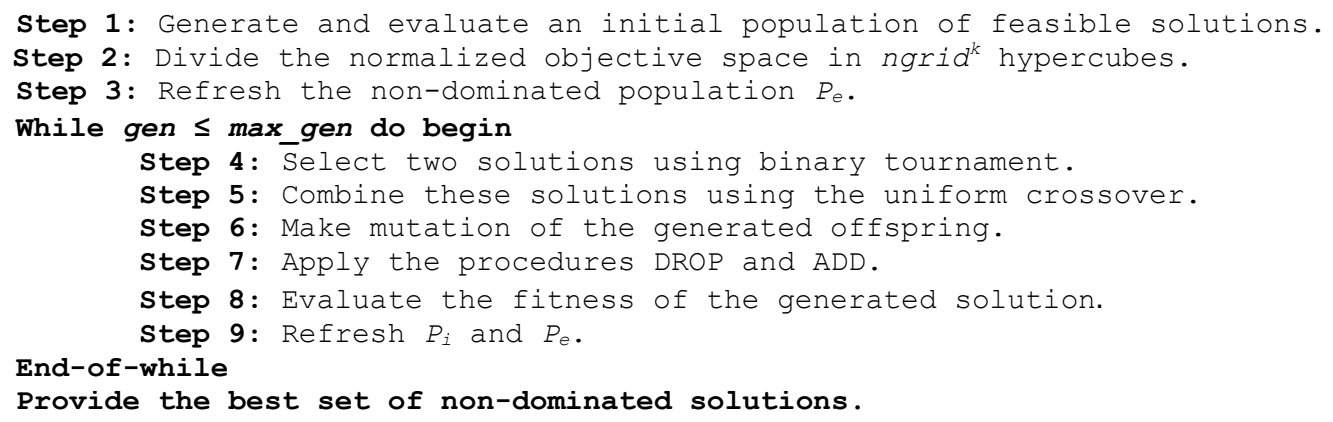

In the proposed algorithm a binary encoding is adopted. Consider a vector of dimension $n$, with $n$ being the number of columns of the problem (or the number of generated duties): if $x_{j}=1$ the column $j$ will be part of the solution; being 0 otherwise.

Each element of the initial population is generated by the constructive phase of the GRASP algorithm (Resende and Ribeiro, 2008) in a reactive procedure (Prais and Ribeiro, 2000). It can be observed that only feasible solutions are generated by the constructive procedure. The evaluation of a given solution is based on the two objective functions, expressed by the Equations (1) and (2). 
The formation of hypercubes is controlled by a parameter ngrid which defines the number of subdivisions of the axis in the space of the objective functions. This space must be bounded and normalized by the minimum and maximum values of the analyzed objective functions.

The population with dominated solutions is named $P_{i}$ and the population with non-dominated solutions is named $P_{e}$. After generating an initial population, a subpopulation is created with non-dominated solutions, which is refreshed in each generation of the algorithm. The selection is performed based on hypercubes. In each generation of the search, a new offspring is generated, which suffers mutation. Then, the improvement operators are applied (repair heuristics and insertion of new duties, when possible). Finally, the populations $P_{i}$ and $P_{e}$ are refreshed.

The selection procedure for the parents crossover is performed by a binary tournament with variable characteristics, which are related with the density of the hypercubes.

In the proposed algorithm, the parents to be selected are not solutions, but hypercubes. The fittest hypercubes, in other words, the hypercubes with lower values of density, will be selected as parents for the crossover operation. The density of a given hypercube is its number of non-dominated solutions. After the selection of a hypercube, a solution that is contained on it is randomly selected.

The selection will be made by the traditional binary tournament when there are four or more hypercubes with a density greater than or equal to 1 . In this case, four hypercubes are randomly selected and a binary tournament is performed.

When the amount of hypercubes with densities greater than or equal to 1 is less than 4 , situation which usually occurs at the early generations of the proposed evolutionary algorithm, the procedure described as follows is performed. Two hypercubes are randomly selected for a tournament. After that operation, a solution of the winner hypercube is randomly selected. This solution will be combined by crossover with a solution contained in $P_{i}$, which is randomly selected.

In the case which there is one hypercube with density greater than or equal to 1 , a solution in this hypercube is selected randomly. Subsequently, it is randomly selected a solution contained in $P_{i}$.

The crossover operator applied in the proposed approach is the uniform crossover with a probability of $100 \%$. Therefore, in each generation of the search, a new solution is generated.

The mutation operator is described as follows: a random number is generated and, if this value is less than or equal the mutation probability, a random bit is selected and inverted, i.e., if the bit is 1 , it will be changed to 0 and vice-versa.

Although the individuals from the initial population are surely feasible, this feasibility will most likely be violated after the crossover and mutation operators have been applied. Hence, we need to reverse this infeasibility and try to improve the corrected solution. Accordingly, the improvement procedures DROP and ADD proposed for the Set Partitioning Problem by Beasley and Chu (1998) were adapted for the maximum coverage problem.

\section{Case study}

Portimão is a city located in the south region of Portugal, with approximately 55 thousands inhabitants. Its economy is based on tourism and since 2003 an urban transportation system was implemented in the abovementioned city.

The main characteristics of the vehicle and crew scheduling in Portimão are described as follows. (i) Pull-in and pull-out trips are allocated in a park of the city. Therefore, we have a single depot problem. (ii) The deadhead times are not available, being considered an average time of 20 minutes. (iii) The maximum waiting time for a vehicle is 15 minutes. (iv) The duties are performed in two or three stages with times varying between one hour to four and a half hours. (v) There is an obligatory meal break. (vi) The breaks are performed between the first and the third hour of the duty, with a total time of three hours. (vii) The changeovers are enabled after a break or in the beginning of a stage. (viii) The daily work time is 8 hours and can reach 14 hours with occurrence of overtime. The main features of the instance are: (i) 401 piece-of-works $(m)$, (ii) 22706 duties $(n)$ and maximum number of duties $(d)$.

The parameters used in the proposed PESA-II are presented below: (i) parameter of the GRASP construction phase ( $\alpha$ ): 100, (ii) size of the population: 50, (iii) number of generations: 500, (iv) crossover probability: 100\%, (v) mutation probability: 100\%, (vi) grid spacing (ngrid): 25 . These parameters were empirically fitted after several computational experiments, enabling a good performance of the suggested metaheuristic.

The proposed PESA-II was implemented in MATLAB, and the experiments were performed on a Genuine Intel $1.86 \mathrm{GHz}$ processor with $1 \mathrm{~GB}$ of RAM memory.

Because of the large dimension of the problem, expressed by the high number of possible duties, the computational times are highly influenced by the parameters size of population and number of generations. Due to the high computational times, a statistical evaluation of the proposed approach was not performed, being considered the results obtained by a single run.

Initially it was used a greedy randomized construction as a mechanism for the generation of the initial population. However, according to the size of the problem, it can be observed that the Genetic Algorithm has presented a difficulty of spreading solutions in an approximation to the Pareto front.

Aiming to increase the Pareto front's fitting quality, it was proposed a hybridization of the metaheuristic method with an exact method, in this case Mixed Integer Programming. The problem with a single objective function (1) was run and solved to optimality in LINGO solver by the Branch-and-Bound (B\&B) method, being inserted in the initial population. The solution obtained by the $B \& B$ is the better solution considering the objective function (1), therefore these solution is an extreme point of the Pareto front. As the several solutions generated in the initial population have not the same quality of the optimal solution, these individual will be isolated in a hypercube. Given the premises of the PESA-II, this hypercube will have a greater probability of selection, and, as a consequence, this solution will have a greater probability of be used in crossover operations. Thus, the good features of the optimal solution for objective function (1) will tend to be transferred to the next generations, incurring in a best fit of the Pareto front. The approximations to the Pareto front are named PESA-II and MP_PESA-II, being presented in the Figure 2.

Visually, it can be observed that the MP_PESA-II approximation is better than the PESA-II approximation. The MP_PESAII approximation to the Pareto front is better distributed, while the PESA-II approximation has a discontinuity in the central region. 
Aiming to evaluate the quality of the generated solutions, we are using three performance indicators: (i) amount of nondominated solutions $|\mathrm{Q}|$; (ii) computational times (t); and (iii) hypervolume (HV). The hypervolume measures the volume of a given dominated portion of the objective space (Deb, 2001). Usually, the grater is the hypervolume, the better is the approximation to the Pareto front.

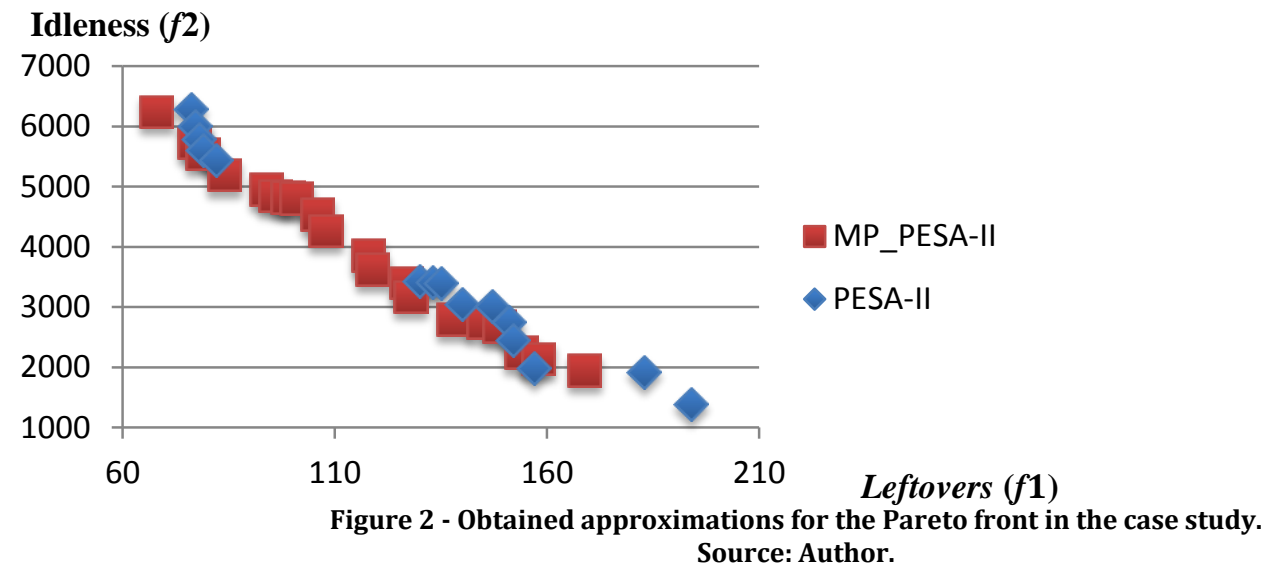

In the Table 1, the computational results are presented for one run of the two tested approaches in the observed case study. On the basis of achieved results, it is worthy of note that MP_PESA-II has presented a greater amount of nondominated solutions than the PESA-II, although the second has presented a better hypervolume.

Table 1: Comparison between PESA-II and MP_PESA-II.

\begin{tabular}{l|l|l}
\hline \multicolumn{1}{c|}{ Indicator } & PESA-II & MP_PESA-II \\
\hline$|\mathrm{Q}|$ & 15 & 20 \\
\hline $\mathrm{t}(\mathrm{s})$ & 5852 & 5738 \\
\hline $\mathrm{HV}$ & 2512710 & 2474946 \\
\hline
\end{tabular}

In the observed case study, there are no available real data concerning the real scheduling performed. Therefore, an accurate comparison between the proposed approach and the real practice was not possible. Although the real scheduling was not available, in according to the author experience, the evolutionary algorithm was able to generate solutions which can be implemented in the real system.

The planners have to generate the scheduling manually, which is a difficult and low flexible process. The generation of a manual scheduling may require some weeks of planning. This laborious process hinders the evaluation of several solutions, leading to solutions which can be improved. The proposed approach can generate a scheduling, considering multiple objective, in less than two hours and better solutions can be achieved increasing the number of generations. Therefore, a great contribution of the proposed approach is the reduction of planning times. Since the process is automated by a parameterized algorithm, it can be used by the planners of the sector.

\section{Conclusions}

The main goal of this work was demonstrating the potential of multiobjective heuristics in solving the Vehicle and Crew Scheduling Problem. In order to obtain an approximation to the Pareto front, hybrid algorithms based on the evolutionary metaheuristic PESA-II were developed.

With regard to case study, unfortunately, there are no available practical solutions made by the planners. Therefore, the comparison between practical solutions and proposed approach is difficult. Because of the size of the instance, the number of iterations of the evolutionary algorithms was only 50 generations. The comparison between the real and computer generated solutions, as well as executing tests with higher number of generations are topics for future studies. However, the obtained results were methodologically consistent. In addition, the benefit of automation in generating solutions is an important feature of the proposed approach.

In conclusion, it can be stated that a multiobjective approach, as this paper presented, propitiates a better and more flexible planning, in sense of offer to decision makers the possibility of choosing solutions in a set of alternatives, without the necessity of impose weights for the criteria in analysis.

\section{References}

Ball, M., Bodin, L. \& Dial, R. (1983) A matching based heuristic for scheduling mass transit crews and vehicles. Transportation Science, $17,4-31$.

Bartodziej, P., Derigs, U., Malcherek, D. \& Vogel U. (2009) Models and algorithms for solving combined vehicle and crew scheduling problems with rest constraints: an application to road feeder service planning in air cargo transportation. OR Spectrum, 31, 405429.

Beasley, J. E. \& Chu, P.C. (1998) Constraint handling in genetic algorithms: the set partitioning problem. Journal of Heuristics, $11,323-357$.

Corne, D. W., Knowles, J. D. \& Oates, M. J. (2000) The Pareto Envelope-based selection algorithm for multiobjective optimization". Proceedings of sixth International Conference on parallel problem solving from Nature, Paris.

Corne, D. W., Jerram, N. R., Knowles, J. D. \& Oates, M. J. (2001) PESA-II: Region-based selection in evolutionary multiobjective optimization. Proceedings of the Genetic and Evolutonary Computation Conference (GECCO - 2001), San Francisco. 
Daduna, J. R. \& Paixão, J. M. P. (1995) Vehicle Scheduling for public mass transit - an Overview. In: Daduna, J. R., Branco, I. e Paixão, J. M. P. (Eds.) Computer-Aided Transit Scheduling, Lecture Notes in Economics and Mathematical Systems, Springer, vol. 430, pp. 76-90.

Deb, K. (2001) Multi-objective optimization using evolutionary algorithms. Chichester: John Wiley \& Sons.

Falkner, J. C. \& Ryan, D. M. (1992) Express: Set partitioning for bus crew scheduling in Christchurch. In: Desrochers, M. e Rosseau, J. M. (Eds.) Computer-Aided Transit Scheduling: Proceedings of the Fifth International Workshop, Springer, 359-378.

Fischetti, M., Lodi, A., Martello, S. \& Toth, P. (2001) A polyhedral approach to simplified crew scheduling and vehicle scheduling problems. Management Science, 47(6), 833-850.

Freling, R., Wagelmans, A. P. M. \& Paixão, J. M. P. (1999) An overview of models and techniques for integrating vehicle and crew scheduling. In: Wilson, N. H. M. (Ed.) Computer-aided Transit Scheduling, Lecture Notes in Economics and Mathematical Systems, Springer, 471, 441-460.

Freling, R., Huisman, D. \& Wagelmans, A.P.M. (2001) Applying an integrated approach to vehicle and crew scheduling in practice. In: Vo $\beta$, S. \& Daduna, J. R. (Eds.) Computer-aided Transit Scheduling, Lecture Notes in Economics and Mathematical Systems, Springer, 505, 73-90.

Freling, R., Huisman, D. \& Wagelmans, A. P. M. (2003) Models and algorithms for integration of vehicle and crew scheduling. Journal of Scheduling, 6, 63-85.

Friberg, C. \& Haase, K. (1999) An exact branch and cut algorithm for the vehicle and crew scheduling problem. In: Wilson, N. H. M. (Ed.) Computer-aided Transit Scheduling, Lecture Notes in Economics and Mathematical Systems, Springer, 471, 63-80.

Gaffi, A. e Nonato, M. (1999) An integrated approach to ex-urban crew and vehicle scheduling problem. In: Wilson, N. H. M. (Ed.) Computeraided Transit Scheduling, Lecture Notes in Economics and Mathematical Systems, Springer, 471, 103-128.

Groot, S. W. \& Huisman, D. (2008) Vehicle and crew scheduling: solving large real-world instances with an integrated approach. In Hickman, M. Mirchandani \& Voß, S. (Eds.) Computer-aided Transit Scheduling, Lecture Notes in Economics and Mathematical Systems, Springer, 600, 43-56.

Haase, K., Desaulniers, G. \& Desrosiers, J. (2001) Simultaneous vehicle and crew scheduling in urban mass transit systems. Transportation Science, 35, 286-303.

Huisman, D., Freling, R. \& Wagelmans, A.P.M. (2005) Multiple-depot integrated vehicle and crew scheduling. Transportation Science, 39, 491-5025.

Kliewer, N., Amberg, B. \& Amberg, B. (2012) Multiple depot vehicle and crew scheduling with time windows for scheduled trips". Public Transport, 3, 213-244.

Laurent, B. e Hao, J.K. (2007) Simultaneous vehicle and driver scheduling: a case study in a limousine rental company. Computers \& Industrial Engineering, 53, 542-558.

Mesquita, M. \& Paias, A. (2008) Set partitioning/covering-based approach for the integrated vehicle and crew scheduling problem. Computers \& Operations Research, 35, 1562-1575.

Mesquita M, Paias A \& Respicio A (2009) Branching approaches for integrated vehicle and crew scheduling. Public Transport, 1, $21-37$.

Patrikalakis, G. \& Xerokostas, D. (1992) Experimentation with a new decomposition scheme of the urban public transport scheduling. In: Desrochers, M. \& Rosseau, J. M. (Eds.) Computer-Aided Transit Scheduling: Proceedings of the Fifth International Workshop, Springer, 407-425.

Prais, M. \& Ribeiro, C. C. (2000) Reactive GRASP: An application to a matrix decomposition problem in TDMA traffic assignment. INFORMS Journal on Computing, 12, 164-176.

Resende, M. G. C. \& Ribeiro, C. C. (2008) Greedy Randomized Adaptive Search Procedures: advances and applications. In: Gendreau, M. e Potvin, J. Y. (Eds.) Handbook of metaheuristics, Kluwer Academic Publishers, pp. 219-250.

Rodrigues, M. K., Souza, C.C. \& Moura, A.V. (2006) Vehicle and crew scheduling for urban bus lines. European Journal of Operational Research, 39, 491-502.

Steinzen, I., Becker, M. \& Suhl, L. (2007) A hybrid evolutionary algorithm for the vehicle and crew scheduling problem in public transit. In: 2007 IEEE Congress on Evolutionary Computation - CEC, Singapore.

Steinzen, I.; Ginter, V.; Suhl, L. \& Kliewer, N. (2010) A Time-Space Network Approach for the Integrated Vehicle and Crew Scheduling Problem with Multiple Depots. Transportation Science, 44, 367-382.

Valouxis, C. \& Housos, E. (2002) Combined bus and driver scheduling. Computers and Operations Research, 170, 844-862.

Wren, A. \& Gualda, N. D. F. (1999) Integrated Scheduling of Buses and Drivers. In: Wilson, N. H. M. (Ed.) Computer-aided Transit Scheduling, Lecture Notes in Economics and Mathematical Systems, Springer, 471, 155-176.

Wren, A. \& Rosseau, J. M. (1995) Bus Driver Scheduling - an Overview. In: Daduna, J. R., Branco, I. \& Paixão, J. M. P. (Eds.) Computer-aided Transit Scheduling, Lecture Notes in Economics and Mathematical Systems, Springer, 430, 173-183. 